\title{
On Some Functions Involving the lcm and ged of Integer Tuples
}

\author{
O. Bagdasar
}

\begin{abstract}
In this paper an explicit formula for the number of tuples of positive integers having the same lowest common multiple $n$ is derived, and some of the properties of the resulting arithmetic function are analyzed. The tuples having also the same greatest common divisor are investigated, while some novel or existing integer sequences are recovered as particular cases. A formula linking the gcd and lcm for tuples of integers is also presented.
\end{abstract}

Keywords: divisibility, lowest common multiple, greatest common divisor, integer sequences

\section{Introduction}

The least common multiple of two natural numbers $a$ and $b$, is usually denoted by $\operatorname{lcm}(a, b)$ or $[a, b]$, and is the smallest number divisible by both $a$ and $b[5, \S 5.1, \mathrm{p} .48]$. The dual notion is the greatest common divisor, denoted by $\operatorname{gcd}(a, b)$ or $(a, b)$ which is the largest number that divides both $a$ and $b$. The lcm and gcd can also be defined for any $k$-tuple of natural numbers $a_{1}, \ldots, a_{k}$, where $k \geq 2$.

For $n=p_{1}^{n_{1}} p_{2}^{n_{2}} \ldots p_{r}^{n_{r}}$, the number of ordered pairs $(a, b)$ having the same $1 \mathrm{~cm} n$ is

$$
|\{(a, b): \operatorname{lcm}(a, b)=n\}|=\left(2 n_{1}+1\right)\left(2 n_{2}+1\right) \cdots\left(2 n_{r}+1\right),
$$

where $\|$ is the cardinality of a set [10]. If $n$ is square-free we have $3^{\omega(n)}$ [4, ex. 2.4, p.101]), where $\omega(n)$ denotes the number of prime divisors of $n$.

The number of relatively prime ordered pairs $(a, b)$ having the same $1 \mathrm{~cm} n$ is (see [8])

$$
|\{(a, b): \operatorname{gcd}(a, b)=1, \operatorname{lcm}(a, b)=n\}|=2^{\omega(n)} .
$$

A property linking the $1 \mathrm{~cm}$ and gcd of the integer pair $(a, b)$ is (see [5])

$$
\operatorname{gcd}(a, b) \operatorname{lcm}(a, b)=a b .
$$

The aim of this paper is to extend the above results to general tuples of integers.

\footnotetext{
Manuscript received March 21 ; accepted June 28

O. Bagdasar is with the School of Computing and Mathematics, University of Derby, Kedleston Road, Derby DE22 1GB, England, U.K., o.bagdasar derby.ac.uk
} 
The number of $k$-tuples of positive integers with the least common multiple $n$ is

$$
\operatorname{LCM}(n ; k)=\left|\left\{\left(a_{1}, \ldots, a_{k}\right): \operatorname{lcm}\left(a_{1}, \ldots, a_{k}\right)=n\right\}\right|,
$$

and some identities and inequalities involving the above arithmetic function are presented. A few sequences indexed in the On-Line Encyclopedia of Integer Sequences (OEIS) [7], are obtained as particular cases.

The number of ordered $k$-tuples with the same $\operatorname{gcd} d$ and $1 \mathrm{~cm} n$ is defined by

$$
\operatorname{GL}(d, n ; k)=\left|\left\{\left(a_{1}, \ldots, a_{k}\right): \operatorname{gcd}\left(a_{1}, \ldots, a_{k}\right)=d, \operatorname{lcm}\left(a_{1}, \ldots, a_{k}\right)=n\right\}\right|,
$$

and a number of properties of this function are analyzed. In the process, some families of integer sequences not currently indexed in the OEIS are produced.

Finally, a property linking the $1 \mathrm{~cm}$ and gcd for $k$-tuples of integers which generalizes formula (3) is presented, accompanied by a brief proof based on [9].

\section{Main results}

In this section the proofs for the formulae of LCM and GL are provided, along with a result linking the $\mathrm{lcm}$ and gcd computed for $k$-tuples of integers.

\subsection{Tuples of integers with the same lcm}

This section presents the formula for the number of ordered $k$-tuples whose least common multiple is a natural number $n$. Some of the properties of this function are then investigated.

Theorem 2.1 Let $k$ and $n$ be naturals numbers. If $n$ has the factorization $n=p_{1}^{n_{1}} p_{2}^{n_{2}} \ldots p_{r}^{n_{r}}$, the number of ordered $k$-tuples whose $1 \mathrm{~cm}$ is $n$ is given by the formula

$$
\operatorname{LCM}(n ; k)=\prod_{i=1}^{r}\left[\left(n_{i}+1\right)^{k}-n_{i}^{k}\right]
$$

Proof. Let $\left\{a_{1}, \ldots, a_{k}\right\}$ be a k-tuple satisfying $\left[a_{1}, \ldots, a_{k}\right]=n$. The numbers $a_{1}, \ldots, a_{k}$ need to be divisors of $n$, therefore they can be factorized as

$$
a_{j}=p_{1}^{a_{1 j}} \cdot p_{2}^{a_{2 j}} \ldots \cdot p_{r}^{a_{r j}}, \quad j=1, \ldots, k,
$$

with $0 \leq a_{i j} \leq n_{i}(i=1, \ldots, r)$. Because their lcm is $n$, one needs to have

$$
\max \left\{a_{i 1}, \ldots, a_{i k}\right\}=n_{i}
$$

for each divisor $p_{i}, i=1, \ldots, r$.

For each $i \in\{1, \ldots, r\}$, the total number of pairs $0 \leq a_{i j} \leq n_{i}(j=1, \ldots, k)$ is $\left(n_{i}+1\right)^{k}$. On the other hand, the number of ordered pairs $0 \leq a_{i j} \leq n_{i}-1(j=1, \ldots, k)$ not having $n_{i}$ as a maximum is $n_{i}^{k}$. The number of pairs satisfying relation (8) is therefore $\left(n_{i}+1\right)^{k}-n_{i}^{k}$. Multiplying this formula for $i=1, \ldots, r$ one obtains (6). 
Remark 2.1 For particular values of $k$, one recovers the following OEIS indexed integer sequences: $\mathrm{A} 048691$ for $\operatorname{LCM}(n ; 2)$ (linked to numerous algebraic interpretations), and $\mathrm{A} 070919, \mathrm{~A} 070920, \mathrm{~A} 070921$ for $\operatorname{LCM}(n ; 3), \operatorname{LCM}(n ; 4), \operatorname{LCM}(n ; 5)$, respectively.

The following result illustrates the independence of $\operatorname{LCM}(n ; k)$ on the prime factors.

Corollary 2.1 Let $k$ be a natural number and $n=p_{1}^{n_{1}} p_{2}^{n_{2}} \ldots p_{r}^{n_{r}}, m=q_{1}^{n_{1}} q_{2}^{n_{2}} \ldots q_{r}^{n_{r}}$, such that all numbers $p_{1}, \ldots, p_{r}, q_{1}, \ldots, q_{r}$ are distinct. Then $\operatorname{LCM}(m ; k)=\operatorname{LCM}(n ; k)$.

Proof. From (6), $\operatorname{LCM}(n ; k)$ only depends on the multiplicities of the prime factors, and not on the prime factors themselves. In this case

$$
\operatorname{LCM}(m ; k)=\prod_{i=1}^{r}\left[\left(n_{i}+1\right)^{k}-n_{i}^{k}\right]=\operatorname{LCM}(n ; k)
$$

Considering relatively prime numbers $m$ and $n$, one obtains the consequence below, which here is proved using a direct method.

Corollary 2.2 Let $m, n$ be integers satisfying $(m, n)=1$. The following property holds

$$
\operatorname{LCM}(m \cdot n ; k)=\operatorname{LCM}(m ; k) \cdot \operatorname{LCM}(n ; k) .
$$

Proof. Consider the following factorizations of $m=q_{1}^{m_{1}} q_{2}^{m_{2}} \ldots q_{s}^{m_{s}}$ and $n=p_{1}^{n_{1}} p_{2}^{n_{2}} \ldots p_{r}^{n_{r}}$. As $(m, n)=1$, it follows that $p_{i} \neq q_{j}$, for any combination of $i=1, \ldots, r$ and $j=1, \ldots, s$. This implies that the prime factorization of the product $m n$ is

$$
m \cdot n=q_{1}^{m_{1}} q_{2}^{m_{2}} \cdots q_{s}^{m_{s}} \cdot p_{1}^{n_{1}} p_{2}^{n_{2}} \cdots p_{r}^{n_{r}},
$$

with the prime factors not necessarily in an increasing order. By formula (6) one obtains

$$
\operatorname{LCM}(m \cdot n ; k)=\prod_{i=1}^{r}\left[\left(m_{i}+1\right)^{k}-m_{i}^{k}\right] \cdot \prod_{j=1}^{s}\left[\left(n_{j}+1\right)^{k}-n_{j}^{k}\right]=\operatorname{LCM}(m ; k) \cdot \operatorname{LCM}(n ; k),
$$

which ends the proof.

Here are recalled some well known properties of arithmetic functions [1, Chapter 2]. An arithmetic function $f(n)$ of the positive integer $n$ is multiplicative if $f(1)=1$ and for any $a$ and $b$ coprime, then $f(a b)=f(a) f(b)$. An arithmetic function is said completely multiplicative if $f(1)=1$ and $f(a b)=f(a) f(b)$, even when $a$ and $b$ are not coprime.

Remark 2.2 From Corollary 2.2, the function $\operatorname{LCM}(n ; k)$ is multiplicative.

Remark 2.3 Let $a, b$ be positive integers and $f: \mathbb{N} \rightarrow \mathbb{N}$ be a multiplicative arithmetic function. The following property holds

$$
f(\operatorname{gcd}(a, b)) \cdot f(\operatorname{lcm}(a, b))=f(a) \cdot f(b) .
$$

Proof. Let $d=\operatorname{gcd}(a, b)$. The numbers $d, a / d$ and $b / d$ are coprime. Using (3) one obtains $f(d) f(\operatorname{lcm}(a, b))=f(d)[f(d) f(a / d) f(b / d)]=[f(d) f(a / d)][f(d) f(b / d)]=f(a) f(b)$.

This ends the proof. 
For $\operatorname{LCM}(n ; k)$ we can check directly the property relative to $1 \mathrm{~cm}$ and $\mathrm{gcd}$.

Corollary 2.3 Let a, b be natural numbers. The following property holds:

$$
\operatorname{LCM}(\operatorname{gcd}(a, b) ; k) \cdot \operatorname{LCM}(\operatorname{lcm}(a, b) ; k)=\operatorname{LCM}(a ; k) \cdot \operatorname{LCM}(b ; k) .
$$

Proof. Assume that the factorizations of $n, d, a, b$ are $n=p_{1}^{n_{1}} p_{2}^{n_{2}} \ldots p_{r}^{n_{r}}, d=p_{1}^{d_{1}} p_{2}^{d_{2}} \ldots p_{r}^{d_{s}}$ and $a=p_{1}^{\alpha_{1}} p_{2}^{\alpha_{2}} \ldots p_{r}^{\alpha_{r}}, b=p_{1}^{\beta_{1}} p_{2}^{\beta_{2}} \ldots p_{r}^{\beta_{r}}$, where some of the powers may be zero. Using the relation (6), it is sufficient to prove the formula for just one fixed value $i$ from $1, \ldots, r$. The contribution of the prime factor $p_{i}$ to formula (11) is

$$
\left[\left(n_{i}+1\right)^{k}-n_{i}^{k}\right] \cdot\left[\left(d_{i}+1\right)^{k}-d_{i}^{k}\right]=\left[\left(\alpha_{i}+1\right)^{k}-\alpha_{i}^{k}\right] \cdot\left[\left(\beta_{i}+1\right)^{k}-\beta_{i}^{k}\right]
$$

This relation is true, as $d_{i}=\min \left\{\alpha_{i}, \beta_{i}\right\}$ and $n_{i}=\max \left\{\alpha_{i}, \beta_{i}\right\}$.

Some inequalities for $\operatorname{LCM}(n ; k)$ can also be proved, for general values of $m$ and $n$.

Theorem 2.2 Let $k, m$ and $n$ be natural numbers. The following inequality holds

$$
\operatorname{LCM}(m \cdot n ; k) \leq \operatorname{LCM}(m ; k) \cdot \operatorname{LCM}(n ; k)
$$

To prove this theorem, we shall first prove the following result.

Lemma 2.3 Let $k \geq 2$, $p$ be a prime number and $\alpha, \beta \geq 1$ natural numbers. Then

$$
\operatorname{LCM}\left(p^{\alpha+\beta} ; k\right) \leq \operatorname{LCM}\left(p^{\alpha} ; k\right) \cdot \operatorname{LCM}\left(p^{\beta} ; k\right)
$$

Proof. The inequality (13) is then equivalent to

$$
\left[(\alpha+\beta+1)^{k}-(\alpha+\beta)^{k}\right] \leq\left[(\alpha+1)^{k}-\alpha^{k}\right] \cdot\left[(\beta+1)^{k}-\beta^{k}\right] .
$$

From (6) we have $\operatorname{LCM}\left(p^{\alpha+\beta} ; k\right)=(\alpha+\beta+1)^{k}-(\alpha+\beta)^{k}=\sum_{l=0}^{k-1}\left(\begin{array}{l}k \\ l\end{array}\right)(\alpha+\beta)^{l}$, then $\operatorname{LCM}\left(p^{\alpha} ; k\right)=(\alpha+1)^{k}-\alpha^{k}=\sum_{i=0}^{k-1}\left(\begin{array}{l}k \\ i\end{array}\right) \alpha^{i}$ and $\operatorname{LCM}\left(p^{\beta} ; k\right)=(\beta+1)^{k}-\beta^{k}=\sum_{j=0}^{k-1}\left(\begin{array}{l}k \\ j\end{array}\right) \beta^{j}$. For a fixed pair $(i, j)$ with $i, j$ chosen from the set $\{0, \ldots, k-1\}$, the term $\alpha^{i} \beta^{j}$ appears in the right-hand side, and its coefficient is $\left(\begin{array}{l}k \\ i\end{array}\right)\left(\begin{array}{l}k \\ j\end{array}\right)$. On the other hand, the term $\alpha^{i} \beta^{j}$ only appears on the left-hand side if $l=i+j \leq k-1$, and its coefficient is $\left(\begin{array}{l}k \\ l\end{array}\right)\left(\begin{array}{l}l \\ i\end{array}\right)$.

To finalize the proof it remains to be shown that the latter coefficient is smaller than the former. One can check that

$$
\frac{\left(\begin{array}{l}
k \\
i
\end{array}\right)\left(\begin{array}{l}
k \\
j
\end{array}\right)}{\left(\begin{array}{l}
k \\
l
\end{array}\right)\left(\begin{array}{l}
l \\
i
\end{array}\right)}=\frac{k(k-1) \cdots(k-i+1)}{(k-j)(k-j-1) \cdots(k-j-i+1)} \leq 1 .
$$

The computational details of the proof are left to the reader as an exercise.

Consider the factorizations of $m=p_{1}^{m_{1}} p_{2}^{m_{2}} \ldots p_{r}^{m_{r}}$ and $n=p_{1}^{n_{1}} p_{2}^{n_{2}} \ldots p_{r}^{n_{r}}$, where $m_{i}, n_{i} \geq 0$. Using Corollary 2.2 for the prime factors $p_{1}, \ldots, p_{r}$ and their powers, and Lemma 2.3 for each of the indices $i=1, \ldots, r$, one obtains (12). This ends the proof of the theorem.

Remark 2.4 It is not difficult to prove that using particular values that the inequality in Theorem 2.2 is strict for $k \geq 2$. For example, for $p=2, \alpha=\beta=1$ one obtains the relation $3^{k}-2^{k}<\left(2^{k}-1^{k}\right)\left(2^{k}-1^{k}\right)$, which is true for all values $k \geq 2$. This also proves that $\operatorname{LCM}(n ; k)$ is not completely multiplicative. 


\subsection{Tuples of integers with the same lcm and ged}

This section evaluates the function $\operatorname{GL}(d, n ; k)$ given by (5), enumerating the $k$-tuples with fixed greatest common divisor $d$ and least common multiple $n$. Some of its properties and particular instances are also presented, along with some novel number sequences.

The following lemma represents the motivation for the results in this section.

Lemma 2.4 Let $d<n$ be positive integers, such that $d \mid n$. The number of ordered pairs $(a, b)$ with the same greatest common divisor $d$ and least common multiple $n$ is

$$
|\{(a, b): \operatorname{gcd}(a, b)=d, \operatorname{lcm}(a, b)=n\}|=2^{\omega(n / d)} .
$$

where $\omega(x)$ represents the number of distinct prime divisors for the integer $x$.

Proof. Let the numbers $d, n, a, b$ have the prime decompositions

$$
d=p_{1}^{d_{1}} p_{2}^{d_{2}} \cdots p_{r}^{d_{r}}, \quad n=p_{1}^{n_{1}} p_{2}^{n_{2}} \cdots p_{r}^{n_{r}}, \quad a=p_{1}^{\alpha_{1}} p_{2}^{\alpha_{2}} \cdots p_{r}^{\alpha_{r}}, \quad b=p_{1}^{\beta_{1}} p_{2}^{\beta_{2}} \cdots p_{r}^{\beta_{r}},
$$

where $1 \leq d_{i} \leq n_{i}$ for $i=1, \ldots, r$.

As $d=(a, b)$ and $n=[a, b], d_{i}=\min \left\{\alpha_{i}, \beta_{i}\right\}$ and $n_{i}=\max \left\{\alpha_{i}, \beta_{i}\right\}$ for each $i \in\{1, \ldots, r\}$. Also, the numbers $a$ and $b$ are distinct, or otherwise $d=n$. There are two possibilities.

When $d_{i}=n_{i}$, one has $\alpha_{i}=\beta_{i}=d_{i}=n_{i}$. Each choice of $i \in I=\left\{i \in\{1, \ldots, n\}: d_{i}<n_{i}\right\}$ generates two possible pairs $\left(\alpha_{i}, \beta_{i}\right) \in\left\{\left(d_{i}, n_{i}\right),\left(n_{i}, d_{i}\right)\right\}$, hence in total there are $2^{|I|}$ distinct pairs of powers. As the prime decomposition of $n / d$ is

$$
n / d=p_{1}^{n_{1}-d_{1}} p_{2}^{n_{2}-d_{2}} \cdots p_{r}^{n_{r}-d_{r}}=\prod_{i \in I} p_{i}^{n_{i}-d_{i}}
$$

one obtains that $|I|=\omega(n / d)$. This ends the proof. For $d=1$ one recovers the result (2), representing the number of unitary divisors, indexed as A034444 in the OEIS.

The following result relates $\operatorname{GL}(d, n ; k)$ to the set of relatively prime tuples, and shows that finding $\operatorname{GL}(d, n ; k)$ can be reduced to evaluating $\operatorname{GL}(1, n / d ; k)$.

Lemma 2.5 Let $k$ and $d \mid n$ be natural numbers. If $d=p_{1}^{d_{1}} p_{2}^{d_{2}} \ldots p_{r}^{d_{r}}$ and $n=p_{1}^{n_{1}} p_{2}^{n_{2}} \ldots p_{r}^{n_{r}}$, the number of ordered $k$-tuples whose $\mathrm{gcd} i s d$, and $\mathrm{lcm}$ is $n$ satisfies the property

$$
\mathrm{GL}(d, n ; k)=\mathrm{GL}(1, n / d ; k) .
$$

Proof. Let $\left\{a_{1}, \ldots, a_{k}\right\}$ be a $k$-tuple satisfying $\left(a_{1}, \ldots, a_{k}\right)=d$ and $\left[a_{1}, \ldots, a_{k}\right]=n$. Numbers $a_{1}, \ldots, a_{k}$ are multiples of $d$ and divisors of $n$, therefore they can be factorized as

$$
a_{j}=p_{1}^{a_{1 j}} \cdot p_{2}^{a_{2 j}} \ldots \cdot p_{r}^{a_{r j}}, \quad j=1, \ldots, k
$$

with $d_{i} \leq a_{i j} \leq n_{i}(i=1, \ldots, r)$. Because their gcd is $d$ and lcm is $n$, one needs to have

$$
\min \left\{a_{i 1}, \ldots, a_{i k}\right\}=d_{i}, \quad \max \left\{a_{i 1}, \ldots, a_{i k}\right\}=n_{i},
$$


for each $p_{i}, i=1, \ldots, r$. The number of tuples in (17) is the same as of the tuples satisfying

$$
\min \left\{a_{i 1}^{\prime}, \ldots, a_{i k}^{\prime}\right\}=0, \quad \max \left\{a_{i 1}^{\prime}, \ldots, a_{i k}^{\prime}\right\}=n_{i}-d_{i},
$$

which relate to the $k$-tuples having the properties $\left(a_{1}^{\prime}, \ldots, a_{k}^{\prime}\right)=1$ and $\left[a_{1}^{\prime}, \ldots, a_{k}^{\prime}\right]=n / d$. This ends the proof.

Lemma 2.6 Let $k$ and $\alpha$ be positive integers. The number of tuples $\left(\alpha_{1}, \ldots, \alpha_{k}\right)$ satisfying

$$
\mathrm{T}(\alpha ; k)=\left|\left\{\left(\alpha_{1}, \ldots, \alpha_{k}\right): \min \left(\alpha_{1}, \ldots, \alpha_{k}\right)=0, \max \left(\alpha_{1}, \ldots, \alpha_{k}\right)=\alpha\right\}\right|
$$

is given by the formula

$$
\mathrm{T}(\alpha ; k)=(\alpha+1)^{k}-2 \alpha^{k}+(\alpha-1)^{k} .
$$

Proof. Consider the term $\mathrm{T}(\alpha ; k+1)$ representing the number of $(k+1)$-tuples $\left(\alpha_{1}, \ldots, \alpha_{k+1}\right)$ whose min is 0 and $\max$ is $\alpha$. There are three distinct cases, depending on the value of $\alpha_{k+1}$.

1. $\alpha_{k+1}=0$. In this case the $k$-tuple $\left(\alpha_{1}, \ldots, \alpha_{k}\right)$ has to satisfy $\max \left(\alpha_{1}, \ldots, \alpha_{k}\right)=\alpha$. Following the argument used in Theorem 2.1, the number of $k$-tuples having this property is $(\alpha+1)^{k}-\alpha^{k}$.

2. $\alpha_{k+1}=n$. Here the restriction on the $k$-tuple $\left(\alpha_{1}, \ldots, \alpha_{k}\right)$ is $\min \left(\alpha_{1}, \ldots, \alpha_{k}\right)=0$. Similarly as above, the number of $k$-tuples with this property is $(\alpha+1)^{k}-\alpha^{k}$.

3. $0<\alpha_{k+1}<n$. For each of the $(\alpha-1)$ possible values of $\alpha_{k+1}$, the $k$-tuples $\left(\alpha_{1}, \ldots, \alpha_{k}\right)$ satisfy $\min \left(\alpha_{1}, \ldots, \alpha_{k}\right)=0$ and $\max \left(\alpha_{1}, \ldots, \alpha_{k}\right)=\alpha$, and their number is $\mathrm{T}(\alpha ; k)$.

By counting all the $(k+1)$-tuples for a fixed $\alpha$, the terms $\mathrm{T}(\alpha ; k)$ satisfy the recurrence

$$
\mathrm{T}(\alpha ; k+1)=2\left[(\alpha+1)^{k}-\alpha^{k}\right]+(\alpha-1) \mathrm{T}(\alpha ; k)
$$

For $\alpha \geq 1$ one has $\mathrm{T}(\alpha ; 0)=\mathrm{T}(\alpha ; 1)=0$, while from the proof of Lemma $2.5, \mathrm{~T}(\alpha ; 2)=2$. Writing the recursion until $\mathrm{T}(\alpha ; 1)$, one obtains

$$
\begin{aligned}
\mathrm{T}(\alpha ; k+1) & =2 \sum_{i=0}^{k}(\alpha-1)^{i}\left[(\alpha+1)^{k-i}-\alpha^{k-i}\right]+(\alpha-1)^{k+1} T(\alpha ; 0) \\
& =2 \sum_{i=0}^{k}\left[(\alpha-1)^{i}(\alpha+1)^{k-i}-(\alpha-1)^{i} \alpha^{k-i}\right] \\
& =2\left[\frac{(\alpha+1)^{k+1}-\alpha^{k+1}}{2}-\frac{\alpha^{k+1}-(\alpha-1)^{k+1}}{1}\right] \\
& =(\alpha+1)^{k+1}-2 \alpha^{k+1}+(\alpha-1)^{k+1},
\end{aligned}
$$

where the relation $a^{k+1}-b^{k+1}=(a-b)\left(a^{k}+a^{k-1} b+\cdots+a b^{k-1}+b^{k}\right)$ valid for any $a, b$ was used. The result proves (19), which could also be checked by mathematical induction. Here the direct proof was preferred, as it shows how the formula can be derived. 
For a simplified notation we may define $\mathrm{L}(n ; k):=\mathrm{GL}(1, n ; k)$, for positive integers $k, n$. The formula for $\mathrm{L}(n ; k)$ now follows as a direct consequence of Lemma 2.6.

Theorem 2.7 Let $k$ and $n$ be naturals numbers. If $n$ has the factorization $n=p_{1}^{n_{1}} p_{2}^{n_{2}} \ldots p_{r}^{n_{r}}$, the number of ordered $k$-tuples whose gcd is 1 and $1 \mathrm{~cm}$ is $n$, is given by the formula

$$
\mathrm{L}(n ; k)=\prod_{i=1}^{r}\left[\left(n_{i}+1\right)^{k}-2 n_{i}^{k}+\left(n_{i}-1\right)^{k}\right] .
$$

Proof. Let $\left\{a_{1}, \ldots, a_{k}\right\}$ be a $k$-tuple satisfying $\operatorname{gcd}\left(a_{1}, \ldots, a_{k}\right)=1$ and $\operatorname{lcm}\left[a_{1}, \ldots, a_{k}\right]=n$. The numbers $a_{1}, \ldots, a_{k}$ can be factorized as

$$
a_{j}=p_{1}^{a_{1 j}} \cdot p_{2}^{a_{2 j}} \ldots \cdot p_{r}^{a_{r j}}, \quad j=1, \ldots, k
$$

with $0 \leq a_{i j} \leq n_{i}(i=1, \ldots, r)$. Because their gcd is 1 and $1 \mathrm{~cm}$ is $n$, one needs to have

$$
\min \left\{a_{i 1}, \ldots, a_{i k}\right\}=0, \quad \max \left\{a_{i 1}, \ldots, a_{i k}\right\}=n_{i},
$$

for each $p_{i}, i=1, \ldots, r$, which from Lemma 2.6 produces $\left(n_{i}+1\right)^{k}-2 n_{i}^{k}+\left(n_{i}-1\right)^{k}$ tuples. By taking the product over all factors $p_{i}, i=1, \ldots, r$ one obtains (21).

The following properties of $\mathrm{L}(n ; k)$ are direct consequences of formula (21), similar to the results obtained for $\operatorname{LCM}(n ; k)$. For this reason these are presented here without proofs. The first property suggests the exclusive dependence of $\mathrm{L}(n ; k)$ on the prime factorization.

Corollary 2.4 Let $k$ be a natural number and $n=p_{1}^{n_{1}} p_{2}^{n_{2}} \ldots p_{r}^{n_{r}}, m=q_{1}^{n_{1}} q_{2}^{n_{2}} \ldots q_{r}^{n_{r}}$, such that all numbers $p_{1}, \ldots, p_{r}, q_{1}, \ldots, q_{r}$ are distinct. Then $\mathrm{L}(m ; k)=\mathrm{L}(n ; k)$.

The following results states the multiplicity of the arithmetic function $\mathrm{L}(n ; k)$ for $k \geq 2$.

Corollary 2.5 Let $m, n$ be comprime integers and $k \geq 2$. The following property holds

$$
\mathrm{L}(m \cdot n ; k)=\mathrm{L}(m ; k) \cdot \mathrm{L}(n ; k) .
$$

The last result is a consequence of the multiplicity.

Corollary 2.6 Let a, b be natural numbers. The following property holds:

$$
\mathrm{L}(\operatorname{gcd}(a, b) ; k) \cdot \mathrm{L}(\operatorname{lcm}(a, b) ; k)=\mathrm{L}(a ; k) \cdot \mathrm{L}(b ; k) .
$$

Remark 2.5 Choosing $m=n=2$ and $k \geq 2$ one may check that

$$
\mathrm{L}(m \cdot n ; k)=3^{k}-2 \cdot 2^{k}+1<\left(2^{k}-2\right) \cdot\left(2^{k}-2\right)=\mathrm{L}(m ; k) \cdot \mathrm{L}(n ; k) .
$$

This proves that $\mathrm{L}(n ; k)$ is not completely multiplicative.

Preliminary investigations suggest that the inequality $\mathrm{L}(m \cdot n ; k) \leq \mathrm{L}(m ; k) \cdot \mathrm{L}(n ; k)$ is true. However, a complete solution is not known to the author. 


\subsection{Integer sequences related to $\mathrm{T}(n ; k)$ and $\mathrm{L}(n ; k)$}

A number of integer sequences can be obtained from $\mathrm{T}(n ; k)$, for particular values of $k$ and $n$. These novel, or existing OEIS number sequences have a unified interpretation, representing the number of ordered $k$-tuples whose minimum is zero and maximum is $n$.

For $n=1$, one obtains $\mathrm{T}(1 ; k)=2^{k}-2$, which is the number of nonempty proper subsets for a set with $k$ elements, indexed as A000918 in the OEIS. For $n=2$, the sequence defined by $\mathrm{T}(2 ; k-1)=3^{k-1}-2^{k}+1$, represents the Stirling numbers of the second kind, indexed as A028243 in the OEIS. The first few terms of the sequences $\mathrm{T}(n ; k)$ for $n=3,4,5$ give

$$
\begin{array}{ll}
\mathrm{T}(3 ; k): & 0,2,18,110,570,2702,12138,52670,223290,931502, \ldots \\
\mathrm{T}(4 ; k): & 0,2,24,194,1320,8162,47544,266114,1448520,7727522, \ldots \\
\mathrm{T}(5 ; k): & 0,2,30,302,2550,19502,140070,963902,6433590,41983502, \ldots
\end{array}
$$

not currently indexed in the OEIS, suggesting that they are probably new number sequences.

For $k=1,2,3$, one obtains $\mathrm{T}(n ; 1)=0, \mathrm{~T}(n ; 2)=2$ and $\mathrm{T}(n ; 1)=3 n$, respectively. For $k=4$, the sequence defined by $\mathrm{T}(n ; 4)=12 n^{2}+2$, represents the number of points on surface of hexagonal prism, indexed as A005914 in the OEIS. Finally, for $k=5$ one obtains $\mathrm{T}(n ; 5)=20 n^{3}+10 n$, which is A068236 (also, $\mathrm{T}(n+1 ; 5)$ produces A101098).

Several integer sequences are obtained from $\mathrm{L}(n ; k)$, for particular values of $k$ and $n$. Some special cases worth mentioning. For a prime number $p$, one has $\mathrm{L}(p ; k)=2^{k}-2$. Also, the values $\mathrm{L}(n ; 1)=0$ and $\mathrm{L}(1 ; k)=1$ are produced, for a positive integer $n$ and $k \geq 2$. If $n=p_{1}^{n_{1}} p_{2}^{n_{2}} \ldots p_{r}^{n_{r}}$, the following results are obtained as particular cases:

$$
\begin{aligned}
& \mathrm{L}(n ; 2)=\prod_{i=1}^{r}\left[\left(n_{i}+1\right)^{2}-2 n_{i}^{2}+\left(n_{i}-1\right)^{2}\right]=2^{\omega(n)}, \\
& \mathrm{L}(n ; 3)=\prod_{i=1}^{r}\left[\left(n_{i}+1\right)^{3}-2 n_{i}^{3}+\left(n_{i}-1\right)^{3}\right]=6^{\omega(n)} \prod_{i=1}^{r} n_{i}, \\
& \mathrm{~L}(n ; 4)=\prod_{i=1}^{r}\left[\left(n_{i}+1\right)^{4}-2 n_{i}^{4}+\left(n_{i}-1\right)^{4}\right]=2^{\omega(n)} \prod_{i=1}^{r}\left[6 n_{i}^{2}+1\right], \\
& \mathrm{L}(n ; 5)=\prod_{i=1}^{r}\left[\left(n_{i}+1\right)^{5}-2 n_{i}^{5}+\left(n_{i}-1\right)^{5}\right]=10^{\omega(n)} \prod_{i=1}^{r}\left[2 n_{i}^{3}+n_{i}\right] .
\end{aligned}
$$

For some particular values of $k$, a number of novel integer sequences can be recovered. For $k=2$, one obtains $\mathrm{L}(n ; 2)=2^{\omega(n)}$, which is as seen before, is A034444 in the OEIS. The first few terms of the number sequences $\mathrm{L}(n ; k)$ for $k=3,4,5$ give

$$
\begin{array}{ll}
\mathrm{L}(n ; 3): & 1,6,6,12,6,36,6,18,12,36,6,72,6,36,36, \ldots \\
\mathrm{L}(n ; 4): & 1,14,14,50,14,196,14,110,50,196,14,700,14,196,196, \ldots \\
\mathrm{L}(n ; 5): & 1,30,30,180,30,900,30,570,180,900,30,5400,30,900,900, \ldots
\end{array}
$$

not currently indexed in the OEIS.

The enumeration of results in this section suggests that some of the sequences presented are new. Also, some existing sequences were given a different interpretation. 


\subsection{A link between the Icm and ged of $k$-tuples of integers}

One can use the prime number factorization to prove the following relations, which link the $1 \mathrm{~cm}$ and gcd of $k$-tuples of integers. Detailed proofs can be found in [9].

Theorem 2.8 Let $k \leq 2$ and $a_{1}, \ldots, a_{k}$ be natural numbers. The following property holds

$$
\operatorname{lcm}\left(a_{1}, a_{2}, \ldots, a_{k}\right)=\frac{\prod_{1 \leq i_{1}<\ldots<i_{u} \leq k} \operatorname{gcd}\left(a_{i_{1}}, \ldots, a_{i_{u}}\right)}{\prod_{1 \leq i_{1}<\ldots<i_{v} \leq k} \operatorname{gcd}\left(a_{i_{1}}, \ldots, a_{i_{v}}\right)},
$$

where $u$ is odd and $v$ is even.

The dual of this theorem can be written as

Theorem 2.9 Let $k \leq 2$ and $a_{1}, \ldots, a_{k}$ be natural numbers. The following property holds

$$
\operatorname{gcd}\left(a_{1}, a_{2}, \ldots, a_{k}\right)=\frac{\prod_{1 \leq i_{1}<\ldots<i_{u} \leq k} \operatorname{lcm}\left(a_{i_{1}}, \ldots, a_{i_{u}}\right)}{\prod_{1 \leq i_{1}<\ldots<i_{v} \leq k} \operatorname{lcm}\left(a_{i_{1}}, \ldots, a_{i_{v}}\right)},
$$

where $u$ is odd and $v$ is even.

Sketch of proof: Assuming that the multiplicities of $p$ in $a_{1}, \ldots, a_{k}$ are $m_{1}, \ldots, m_{k}$, the problem of proving (34) can be reduced to proving the identity below.

$$
\max \left(m_{1}, \ldots, m_{k}\right)=\sum_{1 \leq i_{1}<\ldots<i_{u} \leq n} \min \left(m_{i_{1}}, \ldots, m_{i_{u}}\right)-\sum_{1 \leq i_{1}<\ldots<i_{v} \leq n} \min \left(m_{i_{1}}, \ldots, m_{i_{v}}\right),
$$

where $u$ is odd and $v$ is even.

\section{Discussion}

The research originated in the counting problem solved by the authors in [2]. There, the Horadam sequences with a fixed period were enumerated using a formula involving all the pairs of numbers having the same lcm, given by formula (1).

In this paper an explicit formula for the number of integer $k$-tuples with the same $1 \mathrm{~cm}$ $n$ denoted by $\operatorname{LCM}(n ; k)$, was established. Some properties of this arithmetic function were explored, being shown that $\operatorname{LCM}(n ; k)$ is multiplicative, but not completely multiplicative. The general formula plays a key role in the enumeration of generalized periodic Horadam sequences, characterized in [3]. The integer sequences $\operatorname{LCM}(n ; 2), \operatorname{LCM}(n ; 3), \operatorname{LCM}(n ; 4)$ and $\operatorname{LCM}(n ; 5)$ were found to be indexed in the OEIS.

The function $\operatorname{GL}(d, n ; k)$ counting the $k$-tuples with same gcd $d$ and lcm $n$ was defined. Most of the properties were linked to the arithmetic function $\mathrm{L}(n ; k):=\mathrm{GL}(1, n ; k)$, whose formula was given. Function $\mathrm{L}(n ; k)$ was multiplicative, but not completely multiplicative. Function $\mathrm{T}(n ; k)$, enumerating $k$-tuples whose min is zero and max is $n$ was also analyzed. Novel, or existing OEIS integer sequences were produced from $\mathrm{T}(n ; k)$ and $\mathrm{L}(n ; k)$.

Finally, two dual properties linking the gcd and $1 \mathrm{~cm}$ of $k$-tuples were presented. 


\section{References}

[1] T. M. Apostol, Introduction to Analytic Number Theory, Springer, New York, 1976.

[2] O. BAgDASAR and P. J. LARCOMBE, On the number of complex Horadam sequences with a fixed period, Fibonacci Quart., Vol. 51, 4 (2013), 339-347.

[3] O. Bagdasar and P. J. Larcombe, On the characterization of periodic generalized Horadam sequences, J. Differ. Equ. Appl. (accepted).

[4] R. Crandall and C. Pomerance, Prime Numbers: A Computational Perspective, Springer, New York, 2001.

[5] G. H. Hardy and E. M. Wright An Introduction to the Theory of Numbers, Oxford University Press, Oxford, Fifth edition, 1979.

[6] Octogon Mathematical Magazine, Collection, 1993-2012.

[7] The On-Line Encyclopedia of Integer Sequences, http://oeis.org, OEIS Foundation Inc. 2011.

[8] R. R. SitARAmachandRa and D. Suryanabayana, The number of pairs of integers with L.C.M. $\leq x$, ARCH. MATH., Vol XXI (1970), 490-497.

[9] D. VĂLCAN and O. D. BAGDASAR, Generalizations of some divisibility relations in $\mathbb{N}$, Creative Math. \& Inf., Vol. 18, 1 (2009), 92-99.

[10] F. Zumhing and T. Andreescu, A Path To Combinatorics for Undergraduates, Birkhauser, 2004. 